\title{
On the early evolution of the Galactic halo
}

\author{
N. Prantzos ${ }^{\star}$ \\ Institut d'Astrophysique de Paris, 98bis Bd. Arago, 75014, Paris, France \\ Received 13 January 2003 / Accepted 18 March 2003

\begin{abstract}
It is shown that the low-metallicity tail of the stellar metallicity distribution predicted by simple Outflow models for the Milky Way halo depends sensitively on whether instantaneous recycling is adopted or relaxed. In both cases, current - and still preliminary - data suggest a "G-dwarf problem" for the halo (reminiscent of the local disk). We suggest that the problem can be solved by introducing a (physically motivated) early infall phase. We point out several important implications of such a modification, concerning: the putative Pop. III (super)massive stars, the number of stars expected at very low metallicities, the questions of primary nitrogen and of the dispersion in abundance ratios of halo stars.
\end{abstract}

Key words. Galaxy: abundances - Galaxy: evolution - Galaxy: formation - Galaxy: halo - Galaxy: stellar content

\section{Introduction}

The metallicity distribution (MD) of long-lived stars is one of the most powerful probes of galactic chemical evolution. The MD of the Galactic halo field stars (and globular clusters) is quite different from the one of the local disk. Its peak at a metallicity $[\mathrm{Fe} / \mathrm{H}] \sim-1.6$ (e.g. Ryan \& Norris 1991) points to an effective yield $y_{\mathrm{HALO}} \sim 1 / 40 Z_{\odot}$ for Fe. This has to be compared to the true yield obtained in the solar neighborhood; in the framework of a specific infall model - evolution at constant gas amount, which is a satisfactory approximation to a more realistic treatment - it can be shown analytically that the local $y_{\text {DISK }} \sim 0.9 Z_{\odot}$ (e.g. Binney \& Tremaine 1987). Taking into account that $\sim 2 / 3$ of solar $\mathrm{Fe}$ are produced by SNIa and only $\sim 1 / 3$ by SNII (e.g. Goswami \& Prantzos 2000) it turns out that the true Fe yield of SNII during the local disk evolution was $\sim 1 / 3 Z_{\odot}$ and, consequently, the effective Fe yield of SNII during the halo phase (where they dominated Fe production) was $y_{\text {HALO }} \sim 1 / 13 y_{\text {DISK }}$.

The simplest interpretation of such a difference between the effective yields in the halo and the local disk remains still the one of Hartwick (1976), who suggested that outflow at a rate $F=k S F R$ (where $S F R$ stands for star formation rate) occurred during the halo formation. In the framework of the Simple model of galactic chemical evolution such outflow reduces the true yield $y_{\text {DISK }}$ to its effective value $y_{\text {HALO }}=$ $y_{\text {DISK }}(1-R) /(1+k-R)$ (e.g. Pagel 1997) where $R$ is the returned mass fraction $(R \sim 0.33$ for a Kroupa et al. 1993 stellar IMF between 0.1 and $100 M_{\odot}$ ); the halo data suggest then that the outflow rate was $k=(1-R)\left(y_{\text {DISK }} / y_{\mathrm{HALO}}-1\right) \sim 8$ times the SFR. [Note: This high outflow rate could be interpreted either as gas expulsion after heating by supernova explosions,

\footnotetext{
* e-mail:prantzos@iap.fr
}

or as simple flowing of gas through the system, e.g. towards the Galaxy's bulge].

In this work we focus on the low-metallicity tail of the halo MD, a fossil of the earliest phase of the halo's evolution. First, we show that the predictions of the simple outflow model in the lowest metallicity range $([\mathrm{Fe} / \mathrm{H}]<-3)$ depend crucially on whether the instantaneous recycling approximation is adopted or relaxed; to our knowledge, it is the first time that this effect is shown to be important in the determination of the MD of a galactic system.

The simple outflow model (with or without IRA) explains readily the peak and the overall shape of the halo MD, but it fails to describe the lowest-metallicity part of it, as revealed recently through the ongoing search of very-low-metallicity stars by the "Beers consortium" (Beers 1999; Norris 1999). The, still preliminary, data suggest that the number of stars is lower by factors of $\sim 5-10$ than predicted by the simple outflow model at $[\mathrm{Fe} / \mathrm{H}] \sim-4$; the discrepancy is larger when the IRA is relaxed (as it should be), as we explain in Sect. 2. This failure gave rise to interesting scenarios concerning the physics of the early Galaxy, such as the effects of inhomogeneous early evolution (e.g. Tsujimoto et al. 1999; Oey 2003) or the - often invoked in the past - pre-enrichment by a putative population III of (super-)massive stars (e.g. Bond 1981; Norris 1999 and references therein).

In this work we suggest a minor - and physically plausible - modification to the simple outflow model: as in the solar neighborhood, the assumption of an early infall phase helps to alleviate the discrepancy between theory and observations concerning the very-low-metallicity tail of the MD. In Sect. 2 we discuss the currently available observational data, we explain the differences between IRA and non-IRA concerning the low-metallicity tail of the MD, and we show how the simple 
outflow model fails in that metallicity range. We also present a composite model of early infall + outflow which provides a much better fit to the - still preliminary - data. In Sect. 3 we discuss the implications of such a scenario for the chemical evolution of the halo concerning, in particular, the role of a putative Pop. III (super-)massive stars, the number of stars expected to be found at very low metallicities, the timescales of the halo evolution and the dispersion in element abundance ratios. The forthcoming results of the "HK survey" (see Sect. 2) concerning the low-metallicity tail of the halo MD will definitely allow to check the robustness of the proposed scenario.

\section{Construction of the MW halo by early infall}

Two major surveys of the halo field star MD in the 90ies (Ryan \& Norris 1991; Carney et al. 1996) concluded that i) the overall shape of the observed MD is consistent with the predictions of a simple outflow model à la Hartwick (1976) and ii) that down to the lowest metallicity limit of the surveys $([\mathrm{Fe} / \mathrm{H}] \sim-3)$ the observed number of metal-poor stars is still consistent with the predictions of that model; according to Carney et al. (1996): "... there is no need to invoke enrichment from Pop III stars".

A much more systematic research of extremely metal poor stars is currently performed by $\mathrm{T}$. Beers and collaborators in the framework of the "HK survey", which concerns more than 10000 metal poor stars. Preliminary results, based on more than half of that sample have already been presented in Beers (1999) and Norris (1999). Various biases affect the results for $[\mathrm{Fe} / \mathrm{H}]>-2$, while the sample could be considered as representative below $[\mathrm{Fe} / \mathrm{H}]=-2$ (Beers 1999). In the left part of Fig. 1 the data of Ryan \& Norris (1991) and of the "Beers consortium", i.e. the differential MD $f(Z)=\mathrm{d} N / \mathrm{d} \log Z$ (where $\mathrm{d} \log Z$ stands for $\mathrm{d}[\mathrm{Fe} / \mathrm{H}]$ ) are displayed on a linear scale (upper panel) and on a logarithmic scale (middle panel). Since the "HK survey" data are unaffected by biases only below $[\mathrm{Fe} / \mathrm{H}]=-2$, we plot them only below that metallicity, assuming that at $[\mathrm{Fe} / \mathrm{H}]=-2$ they match the corresponding data of Ryan \& Norris (1991).

Superimposed on the data are the results of three models of galactic chemical evolution. The dashed curves correspond to a pure outflow model with IRA and outflow rate equal to 8 times the SFR, for which an analytical solution can be obtained. The thin solid curves correspond to the same model with IRA relaxed (numerical solution). In both cases, the stellar initial mass function (IMF) is from Kroupa et al. (1993) between $0.1 M_{\odot}$ and $100 M_{\odot}$, the massive star yields from Woosley \& Weaver (1995) and the intermediate star yields from van den Hoek \& Groenewegen (1997); both sets of yields are metallicity-dependent. SNIa are included in the calculation, (see Goswami \& Prantzos 2000) but their role is negligible in the very early halo phase. It can be seen that

i) The simple outflow model (with or without IRA) fits extremely well the overall shape of the MD down to $[\mathrm{Fe} / \mathrm{H}]=-3$ for the combined sample of Norris \& Ryan (1991) and the "Beers consortium" (as noticed in many previous studies);

ii) An increasingly large discrepancy between the IRA and non-IRA cases arises below $[\mathrm{Fe} / \mathrm{H}]=-3$; this is clearly seen in the middle and bottom left panels of Fig. 1, where the differential and cumulative MDs are plotted, respectively, on logarithmic scales. This is one of the main results of this work. It can be easily understood as follows: When IRA is adopted, each stellar generation ejects instantaneously after its formation the totality of its $\mathrm{Fe}$ yield to enrich the intestellar medium. When IRA is relaxed, only part of that yield becomes available early on (i.e. the Fe ejected by the most massive and short-lived stars, say first above $50 M_{\odot}$, then above $30 M_{\odot}$, then above $20 M_{\odot}$, etc.); for a constant IMF, this implies that more stars (of all masses) have to be formed in that case in order to reach the same metallicity as in the case of IRA. Of course, after some time, all first-generation massive stars have evolved and the yield reaches its final value; after that moment the two models converge. In Fig. 1 (left middle panel) that convergence occurs at $[\mathrm{Fe} / \mathrm{H}] \sim-3.5$. In the right bottom panel of Fig. 1 it can be seen that this metallicity is reached after $\sim 35 \mathrm{Myr}$, i.e. the lifetime of $\sim 8 M_{\odot}$ star, the less massive Fe producers among the massive stars. Obviously, it is the more "realistic" non-IRA case that should be compared to observations (but see Sect. 4 for a discussion of that point).

iii) An increasingly large discrepancy with the data appears below $[\mathrm{Fe} / \mathrm{H}]=-3.5$ in both IRA and non -IRA cases, but the discrepancy is even more important in the non-IRA case: It reaches a factor of $\sim 12$ at the lowest metallicity values attained in the HK survey. That discrepancy gave rise to interesting discussions on the nature of the first stellar populations, as mentioned in Sect. 1.

Despite its spectacular success in the metallicity range $-3<$ $[\mathrm{Fe} / \mathrm{H}]<-1$ the pure outflow model is based on a physically implausible assumption (as is the closed box model), namely that the system "waited" until the total amount of gas became available, and only then started forming stars and expelling (part of) that gas. Instead, the idea that star formation started with a low initial amount of gas and the system continued accreting for some time appears much more plausible (see Sect. 3). One can see immediately that this idea helps to alleviate the observed paucity of low metallicity stars, exactly as it helps to cure the G-dwarf problem in the solar neighborhood.

The thick solid curves on the left panels of Fig. 1 present the results of a composite model, including early infall of primordial composition (lasting for about $0.2 \mathrm{Gyr}$ ) and outflow as in the simple model. The form of infall is Gaussian (Fig. 1, middle right panel, dashed curves), with maximum at $0.1 \mathrm{Gyr}$ and FWHM of $0.04 \mathrm{Gyr}$ and is adopted only for illustration purposes. It can be seen that this composite model produces a much smaller number of stars than the simple outflow model (as expected): by a factor of $\sim 20(10)$ at $[\mathrm{Fe} / \mathrm{H}]=-4$ and $\sim 100(12)$ at $[\mathrm{Fe} / \mathrm{H}]=-5$, for non-IRA (IRA in parenthesis) models. Other forms of infall might lead to even larger differences with respect to the simple outflow model, but this one matches relatively well the currently available - and still preliminary - data. Gaussian infall has been adopted in the case of the solar neighborhood in Prantzos \& Silk (1998), but despite its two degrees of freedom (maximum value and $F W H M$ ) it does not produce considerably better fits to the data than the simpler exponential infall model (e.g. Boissier \& Prantzos 1999). When the final results of the HK survey will become available, it will be possible to determine the form of the 

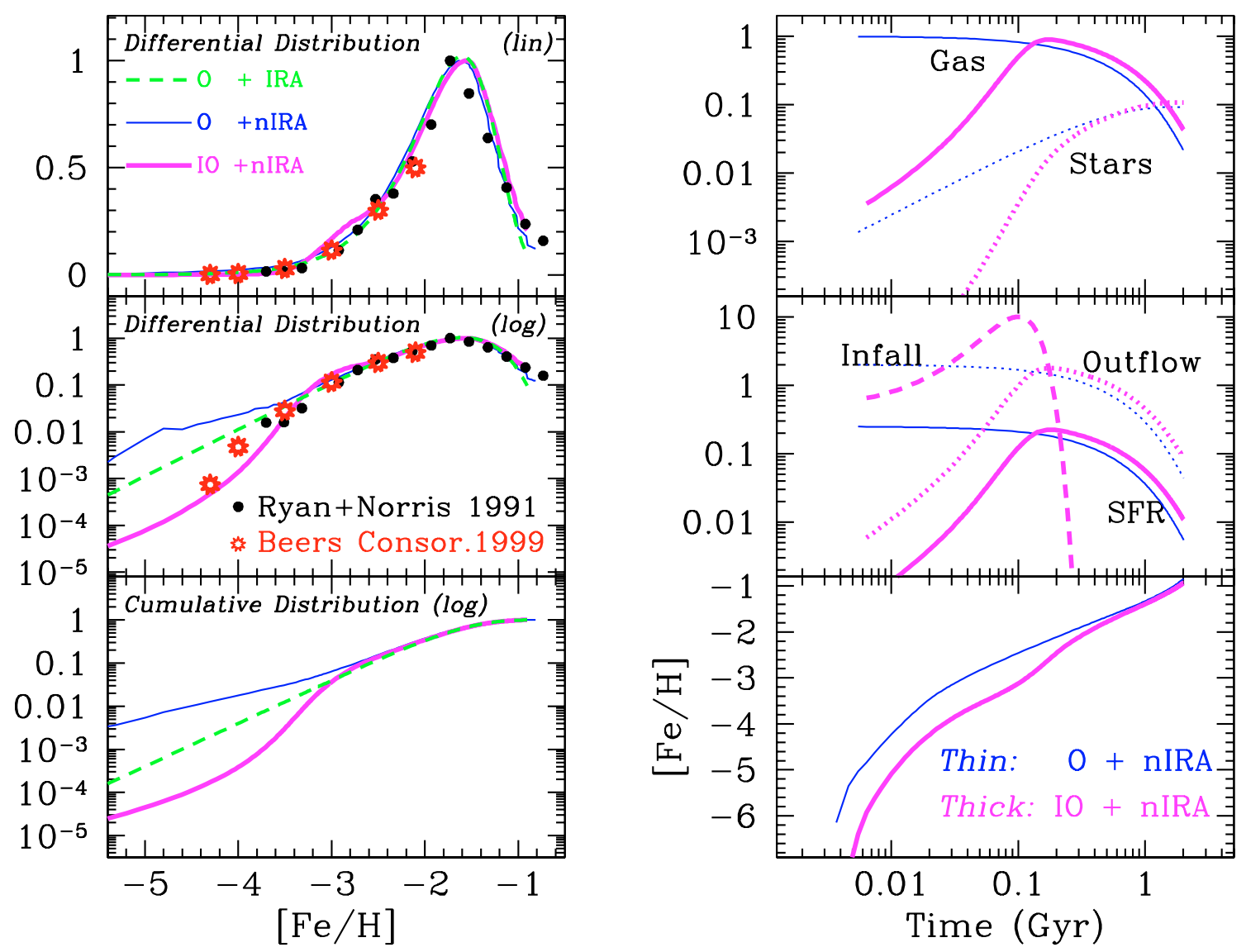

Fig. 1. Halo metallicity distributions (MD, left) and features of relevant models discussed in the text (right). Left top: Differential MD of halo field stars on a linear scale; data points from Ryan \& Norris (1991, filled circles) and the "Beers consortium", as presented in Norris (1999, asterisks); model results for the pure outflow model with IRA (O+IRA, dashed curves), the pure outflow model with IRA relaxed (O+nIRA, thin solid curves) and for the early infall + ouflow model (IO+nIRA, thick solid curves). Left middle: Differential MD of halo field stars on a logarithmic scale; symbols and curves as previously defined. Left bottom: Cumulative MD of halo field stars; model curves as previously defined. Right top: Evolution of gasesous mass (solid curves) and stellar mass (dotted curves) for the pure outflow model with non-IRA (thin curves) and for the early infall + ouflow model (thick curves); all quantities are expressed per unit mass of gas. Right middle: Evolution of SFR (solid curves) and Outflow rate (dotted curves), as well as of the infall rate (dashed curve) for the same models; all quantities are expressed per unit mass of gas and per Gyr. Right bottom: Evolution of $[\mathrm{Fe} / \mathrm{H}]$ in the gas for the same models.

infall rate in the very early Galaxy or, more precisely, the early star formation history of the halo; this will lead to much tighter constraints on future hydrodynamical models of the Milky Way halo formation (see Bekki \& Chiba 2001 for a recent attempt).

Inserting the new ingredient of early infall in the old Outflow "paradigm" seems quite plausible on physical grounds. Indeed, in any system forming stars on a large scale (except, perhaps, in starbursts) an early phase of gas accretion onto a local gravitational potential well seems unavoidable and during that phase a small number of stars may be formed. Outflow due to stellar "feedback", i.e. gas heated by kinetic energy of stellar winds and supernovae, should take place with some time delay, allowing for the collective effects of many of those stellar sources to operate. The model presented in Fig. 1 is meant to simulate just such a sequence of events.

At present, it is impossible to derive timescales for the duration of that sequence of events, based only on the observed MD. The situation is different in the solar neighborhood, where we know: 1) the age of the system to within $\sim 20 \%$ (the age of the local disk is evaluated to be $8-12$ Gyr) and 2) that it reached solar metallicity $4.5 \mathrm{Gyr}$ ago. Combined with the local MD, this information allows us to derive a long timescale of $\sim 7$ Gyr for the formation of the local disk (e.g. Chiappini et al. 1997; Boissier \& Prantzos 1999). Comparable information is not available for the halo, since the duration of its formation is known to less than a factor of two (between one Gyr and 2-3 Gyr).

\section{Implications}

The most important direct implication of the above analysis is that there is no need to invoke a Pop III of zero metal (super-)massive stars in order to explain the observed halo MD; in a similar way, the infall hypothesis makes obsolete the need of a local disk pre-enrichment by the halo stars in order to solve the G-dwarf problem. Of course, the second chemical observable of halo stars, namely abundance ratios with strange behaviour at the lowest metallicities (like $\mathrm{Mn} / \mathrm{Fe}$ or $\mathrm{Cr} / \mathrm{Fe}$, e.g. McWilliam 1997), could still be invoked to justify the existence of Pop III stars (e.g. Umeda \& Nomoto 2002). 
However, current uncertainties in the mecanism of core collapse supernova explosion (e.g. Janka et al. 2003) do not allow us to draw any firm conclusion on the expected yield ratios from those objects at any metallicity (see Chieffi \& Limongi 2002 for a recent analysis of yields of stars of zero initial metallicity). Therefore, to the author's opinion, there is currently no strong observational argument for the existence of (super)massive Pop III stars (or of a very peculiar stellar IMF) preceding the formation of the galactic halo and affecting its early chemical evolution.

A second implication, closely related to the first one, concerns the number of stars expected to be detected at very low metallicities by current or future surveys. The early infall + outflow model presented in Fig. 1 has a fraction of $3 \times$ $10^{-4}$ stars with $[\mathrm{Fe} / \mathrm{H}]<-4$ and $3 \times 10^{-5}$ stars with $[\mathrm{Fe} / \mathrm{H}]<$ -5 (Bottom left panel). These numbers suggest that the current record holder of low $\mathrm{Z}$ stars, namely the giant HE 0107-5240 of $[\mathrm{Fe} / \mathrm{H}]=-5.2$ (Christlieb et al. 2002) may well remain the only of its kind not only at the completion of the HK survey ( 10000 stars) but also in future surveys exploring samples ten times larger. The quest for objects with even lower metallicities will require million-object samples.

Taking into account that the mass of the halo is estimated to $\sim 10^{9} M_{\odot}$ (Morrison 1993), that the Kroupa et al. (1993) IMF has $\sim 2$ stars per unit mass below $0.8 M_{\odot}$, and assuming that the aforementioned infall + outflow model applies to the halo as a whole, one sees that there must be about $5 \times 10^{5}$ stars with $[\mathrm{Fe} / \mathrm{H}]<-4,5 \times 10^{4}$ stars with $[\mathrm{Fe} / \mathrm{H}]<-5$, and even a few thousand stars with $[\mathrm{Fe} / \mathrm{H}]<-6$ (note that for a normal IMF extending down to $0.1 M_{\odot}$, the vast majority of them are red dwarfs). Clearly, if (and that is a big if) the proposed modification to the simple outflow model describes satisfactorily the early halo evolution, there is a great potential for discoveries of large numbers of extremely low Z stars by future (very) large scale surveys, like the GAIA mission (see de Zeeuw 2003 for a summary of the perspectives of GAIA).

A third implication of the above results concerns the timescales of the early halo evolution. In Fig. 1 (Bottom right panel) it can be seen that in the case of the simple outflow model it takes only $35 \mathrm{Myr}$ to reach $[\mathrm{Fe} / \mathrm{H}]=-3$, while it takes almost three times as long $(\sim 100 \mathrm{Myr})$ in the case of the infall + outflow model. In the former case, only massive stars have time to enrich the interstellar medium; in the latter, even stars with $\mathrm{M}>4 M_{\odot}$ have time to evolve up to the AGB phase and eject their products of shell He-burning (C and s-process elements) or of Hot Bottom Burning (HBB), i.e. primary nitrogen.

The answers on several important current problems of galactic chemical evolution may depend on those timescales. For instance, the N/Fe ratio in the Milky Way seems to be constant and $\sim$ solar as a function of $[\mathrm{Fe} / \mathrm{H}]$, from $[\mathrm{Fe} / \mathrm{H}] \sim-3$ up to $[\mathrm{Fe} / \mathrm{H}]=0$. This constancy suggests that $\mathrm{N}$ is produced as a primary element (i.e. with a yield independent on the initial metallicity of the star) quite early in the Milky Way. In the pure outflow model, only massive stars can be invoked for such an early production of primary nitrogen, but all current models of massive star nucleosynthesis produce only secondary $\mathrm{N}$; even the recent massive star models including rotation (Meynet \& Maeder 2002) produce insufficient amounts of primary $\mathrm{N}$ to explain the observations (Prantzos 2003). In the infall + outflow model, intermediate mass stars have time enough to evolve before the metallicity reaches $[\mathrm{Fe} / \mathrm{H}]=-3$ and could naturally produce the required primary nitrogen, either by HBB or by rotational mixing.

A fourth implication of the different timescales obtained in the simple and modified halo models concerns the dispersion in the elemental abundance ratios at low metallicities. From the theoretical point of view, it is expected that mixing of stellar ejecta with the ISM should have been incomplete in the early Galaxy, when the mixing timescale of a few Myr was comparable to the evolutionary timescale of the system and of the first stellar generation (Audouze \& Silk 1995; Tsujimoto et al. 1999; Argast et al. 2000; Ishimaru et al. 2003). However, Carretta et al. (2002) find that, down to the lowest metallicity reached by current surveys $([\mathrm{Fe} / \mathrm{H}] \sim-3)$, dispersion in abundance ratios of intermediate mass elements is no larger than expected from observational uncertainties. The question "When was the metallicity $[\mathrm{Fe} / \mathrm{H}]=-3$ reached during the halo evolution?" becomes obviously important in that context and the answer may depend (also) on the modifications of the simple model discussed here. Note, however, that in the framework of inhomogeneous chemical evolution models Tsujimoto et al. (1999) and Argast et al. (2000) obtain "naturally" much larger timescales than in all the models discussed in Sect. 2.

\section{Summary}

In this work it is shown that the low metallicity tail of the MD predicted by simple Outflow models for the MW halo depends sensitively on whether IRA is adopted or relaxed. In both case, current - and still preliminary - data suggest a "G-dwarf problem" for the halo. We suggest that it can be solved by introducing a physically motivated early infall phase; we adopt here a Gaussian infall rate, which turns out to fit the available data well, but determination of the precise form of the infall will have to wait for the results of complete surveys in the future. We point out several important implications of such a modification, concerning: the putative Pop. III (super)massive stars, the number of stars expected at very low metallicities, the questions of primary nitrogen and of the dispersion in abundance ratios of halo stars. The forthcoming results of the "HK survey" concerning the low metallicity tail of the halo MD, will definitely allow to check the robustness of the proposed scenario.

The phenomenological analysis of this work does not take into account the modern framework of early galaxy formation in a cosmological context. First results of such studies have been recently presented (Bekki \& Chiba 2001) and the issue of the halo MD has been successfully addressed, albeit without a clear physical explanation for that success.

Acknowledgements. I am grateful to the referee, U. Fritze von Alvesleben, for her constructive criticism. 


\section{References}

Argast, D., Samland, M., Gerhard, O., \& Thielemann, F.-K. 2000, A\&A, 356, 873

Audouze, J., \& Silk, J. 1995, ApJ, 451, L49

Beers, T. 1999, in Galaxy Evolution: connecting the distant Universe with the local fossil record, ed. M. Spite (Dordrecht: Kluwer), 105

Bekki, K., \& Chiba, M. 2001, ApJ, 558, 666

Boissier, S., \& Prantzos, N. 1999, MNRAS, 307, 857

Bond, H. E. 1981, ApJ, 248, 606

Binney, J., \& Tremaine, S. 1987, Galactic Dynamics (Princeton University Press)

Carney, B., Laird, J., Latham, D., \& Aguilar, L. 1996, AJ, 112, 668

Carretta, E., Gratton, R., Cohen, J., Beers, T., \& Christlieb, N. 2002, AJ, 124, 481

Chiappini, C., Matteucci, F., \& Gratton, R. 1997, ApJ, 477, 765

Chieffi, A., \& Limongi, M. 2002, ApJ, 577, 281

Christlieb, N., Bessel, M., Beers, T., et al. 2002, Nature, 419, 904

de Zeeuw, T. 2003, in The Dynamics, Structure and History of galaxies, ed. G. da Costa, \& H. Jerjen, ASP Conf. Ser., p. 145

Goswami, A., \& Prantzos, N. 2000, A\&A, 359, 151

Hartwick, F. 1976, ApJ, 209, 418

Ishimaru, Y., Prantzos, N., \& Wanajo, S. 2003, in preparation
Janka, H.-T., Buras, R., Kifonidis, K., Plewa, T., \& Rampp, M. 2003, in From Twilight to Highlight: the physics of supernova explosions, ed. W. Hillebrandt, \& B. Leibundgut, ESO Astrophysics symposia (Berlin: Springer), 39

Kroupa, P., Tout, C., \& Gilmore, G. 1993, MNRAS, 262, 545

Mc William, A. 1997, ARA\&A, 35, 503

Meynet, G., \& Maeder, A. 2002, A\&A, 390, 561

Morrison, H. L. 1993, AJ, 106, 578

Norris, J. 1999, in The Galactic Halo, ed. B. Gibson, T. Axelrod, \& M. Putnam, ASP Conf. Ser., 165, 213

Oey, M. S. 2003, MNRAS, 339, 849

Pagel, B. 1997, Nucleosynthesis and galactic chemical evolution (Cambridge University Press)

Prantzos, N. 2003, in CNO in the Universe, ed. C. Charbonnel, D. Schaerer, \& G. Meynet, ASP Conf. Ser., in press [astro-ph/0301043]

Prantzos, N., \& Silk, J. 1998, ApJ, 507, 229

Ryan, S., \& Norris, J. 1991, AJ, 101, 1865

Tsujimoto, T., Shigeyama, T., \& Yoshii, Y. 1999, ApJ, 519, L63

Umeda, H., \& Nomoto, K. 2002, ApJ, 565, 385

van den Hoek, L., \& Groenewegen, M. 1997, A\&A, 123, 305

Woosley, S., \& Weaver, T. 1995, ApJS, 101, 181 